\title{
Cortical Laminar Necrosis as a Presenting Manifestation of Migraine in an Apparently Normal Patient: A Rare Case Report
}

\author{
Shri Ram Sharma Hussain Massaraf ${ }^{1}$ Sumit Das² \\ ${ }^{1}$ Department of Neurology, North Eastern Indira Gandhi Regional \\ Institute of Health and Medical Science, Shillong, Meghalaya, India \\ ${ }^{2}$ Department of Radiology, North Eastern Indira Gandhi Regional \\ Institute of Health and Medical Science, Shillong, Meghalaya, India
}

Arnab Kalita²

\begin{abstract}
Keywords

- cortical laminar necrosis

- migraine

- migrainous infarct

- stroke

We present a 27-year-old female, a known case of classical migraine headache, who had a severe episode of migraine with visual aura attack which continued late into night. The next morning, she had persistent headache and developed abrupt onset of dysarthria and right hemiparesthesias. She attributed symptoms to her long-lasting headache problem and hence did not seek medical help for the next 2 weeks. The symptoms persisted despite her headache subsiding over the next 36 hours. Her condition worsened 2 weeks later during another such episode of headache. This time she developed right hemiparesis, right hemihypoesthesia, and brief and autolimited left-clonic facial movements. Extensive neurological workup done to rule out other known causes of cerebral infarct with cortical laminar necrosis (CLN) was unrevealing. Magnetic resonance imaging of the brain showed left frontoparietal T2-/T1-/ fluid-attenuated inversion recovery hyperintensity without diffusion restriction in diffusion-weighted imaging and subtle blooming in gradient recalled-echo and was radiologically compatible with cortical laminar necrosis. The patient improved with antiplatelets, antimigraine prophylaxis, and stroke rehabilitation therapy. Our present case is a unique one where CLN can be associated with cerebral ischemic infarct due to migrainous etiology.
\end{abstract}

\begin{abstract}
Address for correspondence Shri Ram Sharma, MBBS, MD, DM, Department of Neurology, North Eastern Indira Gandhi Regional Institute of Medical Sciences (An Autonomous Institute, Ministry of Health and Family Welfare, Government of India), Shillong, Meghalaya, India (e-mail: srmsims_sharma@rediffmail.com).
\end{abstract}

\section{Introduction}

Cortical laminar necrosis (CLN) is a less common form of cortical infarction where there is a selective pan necrosis of the cerebral cortex (involving neurons, glia, and blood vessels while underline white matter is completely or relatively spared). ${ }^{1,2}$ The gray matter of the cortex has six layers. The third layer is the most vulnerable. ${ }^{3}$ It has been reported to be associated with hypoxic encephalopathy, hypoglycemic encephalopathy, immunosuppressive therapy, status epilepticus, and cerebral infarction. It is rarely associated with migrainous infarction.
The appearance of the magnetic resonance images (MRIs) in the setting of cortical laminar necrosis can be deceptive. ${ }^{3}$ On MRI, CLN is characterized by a high-intensity cortical signal on T1-weighted and fluid-attenuated inversion recovery (FLAIR) images, and without signs of hemorrhage which shows a typical curvilinear gyriform distribution, following the cerebral convolutions affected. CLN represents to neuronal ischemia accompanied by gliosis and layered deposition of fat-laden macrophages. ${ }^{1-3}$

We present the case of young women who suffered an unusual case of cerebral infarction secondary to migrainous cerebral infarction manifested as CLN on brain MRI. 


\section{Case Report}

We report a 27-year-old female, a lady from Arunachal Pradesh (India), with a long history of intermittent, throbbing, and moderately severe headache in the left hemicrania that had been present for 7 years. Often, the episode of headache used to be preceded by visual perceptions of bright star-shaped objects "floating" in front of her eyes. Accompanied the headache, nausea, vomiting, photophobia, or phonophobia was also described. The episode of headache usually lasted more than 4 to 6 hours on an average, sometimes 1 to 2 days. She was never on any medication for migraine prophylaxis or triptans for acute episodes of migraine. She recalled her father having episodes of such severe headaches on and off for many years. There was no history of alcohol, nicotine, and narcotics substance abuse. On the morning, before the fateful day, she had a prolonged episode of migraine (preceded by visual aura) which continued into next evening for 36 hours. With ongoing headache, she developed dysarthria along with paresthesias of right half of the body, the day after the onset of her headache. She did not seek medical help for 2 weeks. On experiencing weakness in the right upper and lower limbs after 2 weeks from the primary event, she decided to visit our institute. During a typical migraine attack with visual aura symptomatology, she also developed autolimited left clonic facial movements (lasting for $<1$ minute).

On examination, vitals were normal and her National Institutes of Health Stroke Scale (NIHSS) score was 4, suggestive of minor stroke. Her modified ranking scale (mRS) score was 2 , as she had slight disability such that she was unable to carry out all previous activities but able to look after her own affairs without assistance. The patient also had right upper motor neuron facial paralysis, right pronator drift. She had neither nuchal rigidity nor semiology of meningeal syndrome. She never received triptans or any other drug which might lead to vasoconstriction. Other systems were normal.

Laboratory investigation revealed normal, including chest X-ray, 12-lead electrocardiography, brain computed tomography scan, two-dimensional echocardiography, and the Doppler ultrasonography of carotid vessels, complete hematological screening, routine biochemical profile, urine analysis, serology for syphilis (venereal disease research laboratory), immunologic blood test, including antinuclear antibodies, extractable nuclear antigens, lupus anticoagulant, IgG and IgM anticardiolipin antibodies, rheumatoid factor, basic homeostasis study, and hypercoagulable panel (including protein $\mathrm{C}$ and $\mathrm{S}$ antithrombin III, factor V Leiden's mutation, and homocysteine). There was no history of substance abuse with vasoconstricted properties such as amphetamines or cocaine.

Clinical course was uneventful. The patient responded favorably to antiplatelet medication and antimigraine medications and physiotherapy. A gradual regression of symptom otology was noted, and 1 week after onset, focal neurological deficit was completely recovered $(\mathrm{mRS}=0$, NIHSS $=0$ ).

Brain MRI on day 15 after the migraine attack showed gyral T2/T1/FLAIR hyperintensity involving the left superior parietal lobule with adjacent subcortical white matter ( - Fig. 1). Another focus noted in the left middle frontal gyrus without diffusion restriction in diffusion-weighted imaging and subtle blooming in gradient-recalled echo - Fig. 2). Intracranial MR angiography of all the four vessels was found to be normal and negative for cerebral venous sinus thrombosis. Electroencephalography was nonepileptic form, showing only left hemisphere slowing.

At the $8^{\text {th }}$ and $9^{\text {th }}$-month follow-up, brain MRI revealed that the characteristic cortical high signal intensity on T1 and FLAIR images progressively faded. Hemosiderin due to chronic hemorrhage was never demonstrated. The patients remain entirely asymptomatic. The areas of the brain most susceptible to migrainous infarct vary; however, the lesser degree of lesion can assist in good prognosis.

\section{Discussion}

Migraine headache is a known yet less common cause of stroke in young. Little is known about the exact demographic
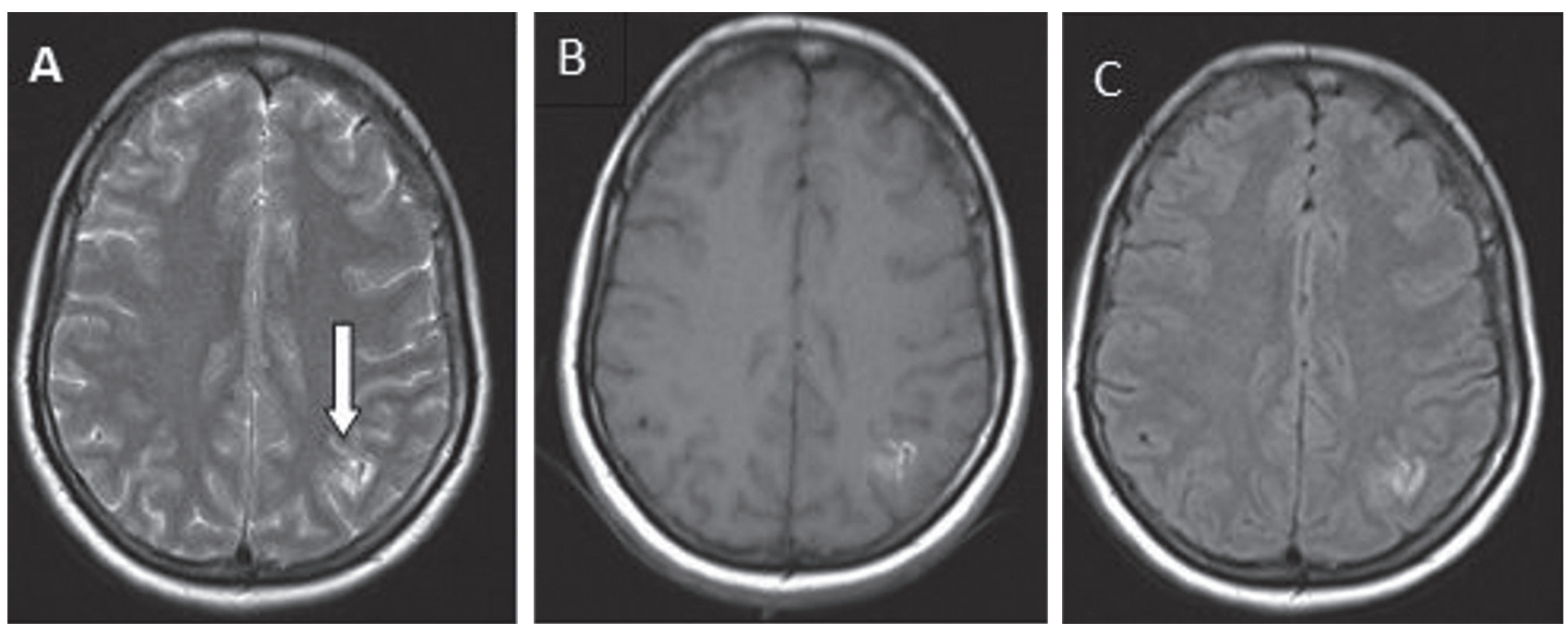

Fig. 1 (A-C) Magnetic resonance imaging at 15 day after ictus: T2-weighted, fluid-attenuated inversion recovery, and T1-weighted sequences show high-intense cortical lesions. 


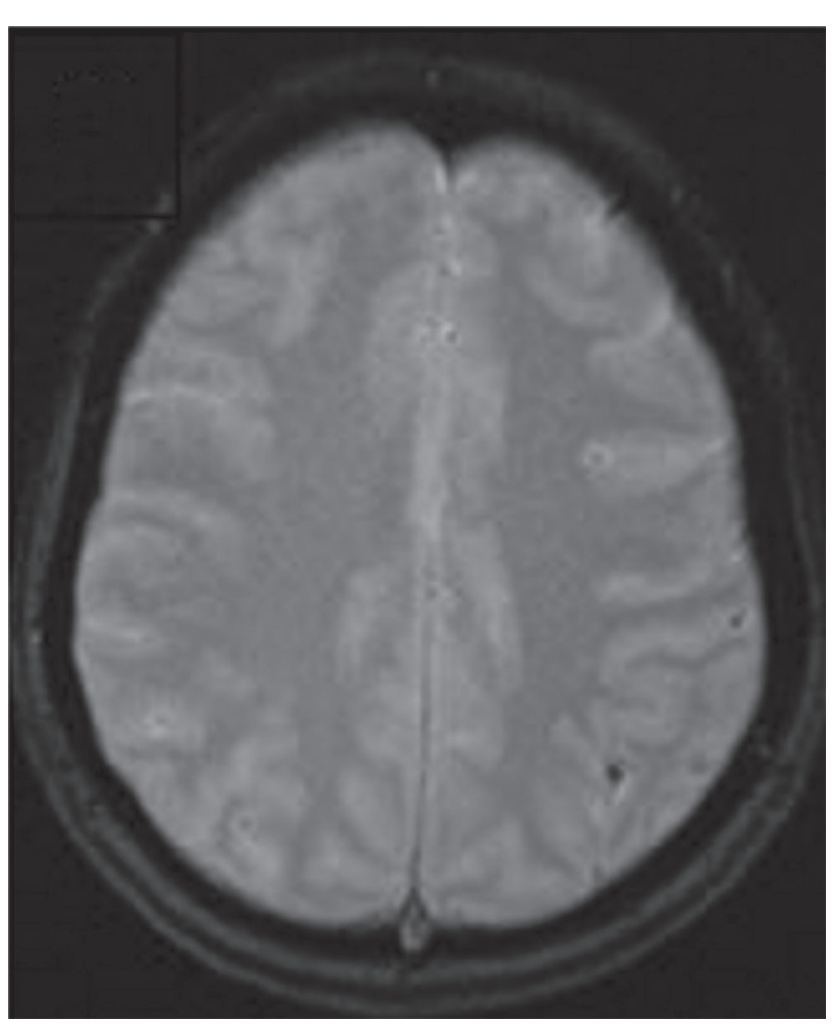

Fig. 2 Gradient echo image shows blooming focus. Magnetic resonance imaging findings suggestive of cortical laminar necrosis.

and statistical association between CLN and migrainous stroke. ${ }^{1,2}$ Ischemic strokes are more common than intracranial bleeds. Classical migraine with aura is more predisposed to vascular insufficiency than those who had migraine without aura. The exact pathophysiology of migrainous infarction is not known. However, some of the possible mechanisms include vascular changes related to cortical spreading depression, vasospasm, and hypercoagulability. ${ }^{2,3}$ Oxidative stress-induced endothelial dysfunction maybe cause and effect of migraine leading to stroke. Potential mimickers for such clinical presentation are mitochondrial encephalopathy, lactic acidosis and stroke-like episodes, Fabry's disease, and arterial dissection. ${ }^{4}$

CLN, as a presenting neuroimaging sign of migrainous stroke, has been infrequently reported. CLN has been associated with cerebral hypoxia, metabolic disturbances, drugs, and infections. CLN usually shows characteristic cortical high intensity on T1-weighted images from 2 weeks to 2 years after ischemia and on FLAIR sequences, hyperintensities appear later in the course of illness. Furthermore, the bloodbrain barrier breakdown due to the necrosis of the blood vessels results in the curvilinear "gyriform" enhancement on gadolinium-enhanced scans typically seen in these cortical infarctions. Hyperintense signal on T1-weighted images can reflect the presence of any of the following substances: hemoglobin, fat, melanin, paramagnetic substances, or protein-rich fluid. ${ }^{56}$ The T1 hyperintensity observed in CLN is not secondary to hemorrhagic transformation of cerebral ischemia and is thought to be due to the selective neuronal damage, followed by higher concentration of proteins (and other macromolecules), glial cell proliferation, and deposition of fat-laden macrophages in the cortical area affected that enhance relatively by restricting the motion of water molecules, thus causing T1 shortening. ${ }^{8}$ Diffusion-weighted MRI shows high intensity of cortical area in brain infarction. Brain diffusion-weighted MRI is a very sensitive tool for detecting neuronal energy depletion and subsequent cytotoxic edema, visualizing such conditions as a high-intensity area. ${ }^{14}$ Excessive release of excitatory amino acids subsequently enhancing calcium influx into cells causes delayed neuronal injury predominantly in the cerebral cortex. In the development of CLN, such neuronal injury produces cytotoxic edema by shrinkage of the extracellular space. CLN is the most likely mechanism for the high signals demonstrated by diffusion-weighted MRI during the subacute period. ${ }^{5}$

To the best of our knowledge, we have found a similar case report of migrainous infarction involving temporoparietal cortical laminar necrosis: the study of Khardenavis et al including a case report of a 27-year-old female with a migrainous infarct with appearance of CLN on MRI and background use of oral contraceptive and heavy smoking were risk factors for migrainous cortical laminar necrosis. ${ }^{7}$ However, she did not have autolimited left clonic facial seizures unlike in the present case which can be explained by the involvement of cerebral cortex related to CLN. ${ }^{10}$

The present case report is compatible with CLN related to migrainous cerebral infarction, as she fulfilled the strict diagnostic criteria for migraine-induced stroke of the International Headache Society which include the following: (1) the patient has previously fulfilled criteria for migraine with aura; (2) the neurological deficit is manifested in the exactly vascular distribution as the aura, persisting for more than 60 minutes, and is associated with an ischemic brain lesion in a suitable territory demonstrated by neuroimaging; and (3) all other potential causes of ischemic stroke with CLN have to be ruled out by appropriate investigations. Migrainous stroke occurs more frequently in women and in patients $<45$ years of age. ${ }^{11-13}$ The patient presents autolimited left clonic facial seizures which can be explained by the involvement of the cerebral cortex related to CLN. Seizures in acute ischemic stroke occur in approximately $5 \%$ of patients and are related to involvement of the cerebral cortex or to very large strokes. ${ }^{9}$

\section{Conclusions}

The present case shows that migrainous infarction is a less common but important cause of stroke in young. Migrainous infarct presents as cortical laminar necrosis and infrequent radiological finding. Vascular changes related to cortical spreading depression, vasospasm, and hypercoagulability are some of the possible mechanisms attributed to cause migrainous infarct.

\section{Funding}

None.

\section{Conflict of Interest}

None declared. 


\section{References}

1 Siskas N, Lefkopoulos A, Ioannidis I, Charitandi A, Dimitriadis AS. Cortical laminar necrosis in brain infarcts: serial MRI. Neuroradiology 2003;45(5):283-288

2 Serrano-Pozo A, González-Marcos JR, Gil-Peralta A. [Cortical laminar necrosis caused by cerebral infarction]. Neurologia 2006;21(5):258-259

3 Sethi NK, Torgovnick J, Macaluso C, Arsura E. Cortical laminar necrosis following anoxic encephalopathy. Neurol India 2006;54(3):327

4 Arboix A, González-Peris S, Grivé E, Sánchez MJ, Comes E. Cortical laminar necrosis related to migrainous cerebral infarction. World J Clin Cases 2013;1(8):256-259

5 Yoneda Y, Yamamoto S. Cerebral cortical laminar necrosis on diffusion-weighted MRI in hypoglycaemic encephalopathy. Diabet Med 2005;22(8):1098-1100

6 Komiyama M, Nakajima H, Nishikawa M, Yasui T. Serial MR observation of cortical laminar necrosis caused by brain infarction. Neuroradiology 1998;40(12):771-777

7 Kinoshita T, Ogawa T, Yoshida Y, Tamura H, Kado H, Okudera T. Curvilinear T1 hyperintense lesions representing cortical necrosis after cerebral infarction. Neuroradiology 2005;47(9):647-651

8 Arboix A, Bechich S, Oliveres M, García-Eroles L, Massons J, Targa C. Ischemic stroke of unusual cause: clinical features, etiology and outcome. Eur J Neurol 2001;8(2):133-139

9 Arboix A, Comes E, García-Eroles L, Massons JB, Oliveres M, Balcells M. Prognostic value of very early seizures for in-hospital mortality in atherothrombotic infarction. Eur Neurol 2003;50(2):78-84

10 Khardenavis V, Karthik DK, Kulkarni S, Deshpande A. Cortical laminar necrosis in a case of migrainous cerebral infarction. BMJ Case Rep 2018;2018:bcr-2017-221483

11 Headache Classification Committee of the International Headache Society. The international classification of headache disorders: 2nd edition. Cephalalgia 2004;24(Suppl 1):9:160

12 Kurth T. Migraine and ischaemic vascular events. Cephalalgia 2007;27(8):965-975

13 Tietjen EG. Migraine and ischaemic heart disease and stroke: potential mechanisms and treatment implications. Cephalalgia 2007;27(8):981-987

14 Bousser MG, Welch KM. Relation between migraine and stroke. Lancet Neurol 2005;4(9):533-542 